\title{
ALMOST PERIODICITY AND CONVERGENT TRIGONOMETRIC SERIES $\left({ }^{1}\right)$
}

\author{
BY \\ ROBERT G. UTTLEY( $\left.{ }^{2}\right)$
}

1. Introduction. Besicovitch [1] has shown that there are infinitely many trigonometric series convergent to any function $f(x)$ bounded and continuous for $-\infty<x<+\infty$ and of bounded variation in every finite interval, and therefore that the sum-function of an everywhere convergent trigonometric series is not necessarily almost periodic. His proof makes clear that almost periodicity of the sum-function can only be ensured by placing a suitable restriction on the exponents of the series. The conditions to be satisfied by the sum-function itself depend on the type of almost periodicity to be established.

By use of the symmetrical (SCP) and unsymmetrical (CP) Cesàro-Perron integrals and the introduction of new distance-functions, $\mathrm{H}$. Burkill [4] has enlarged the class of almost periodic functions sufficiently to enable him to obtain the following result, whose most remarkable feature is that no assumption is made concerning the integrability of the sum-function. It is a generalization of a similar theorem for purely periodic functions, due to J. C. Burkill [5].

H. BURKILl's ThEOREM. If $\left(\lambda_{n}\right)$ be a sequence of real numbers such that

$$
\lambda_{1}>0 \quad \text { and } \quad \lambda_{n+1}-\lambda_{n} \geqq l>0 \quad(n=1,2, \cdots)
$$

and if, for all $x$, the series

$$
a_{0} / 2+\sum_{n=1}^{\infty}\left(a_{n} \cos \lambda_{n} x+b_{n} \sin \lambda_{n} x\right)
$$

converges to $f(x)$, then $f(x)$ is SCP-integrable and SCP-a.p., and this series is the Fourier series of $f(x)$. In particular,

(i) If $f(x)$ is CP-integrable, then it is CP-a.p.

(ii) If $f(x)$ is bounded, then it is D.a.p.

(iii) If $f(x)$ is bounded and uniformly continuous, then it is u.a.p.

Received by the editors October 17, 1960.

(1) The results contained in this paper are essentially part of a thesis submitted to the University of Sheffield, England, in 1951 in partial fulfillment of the requirements for the degree of Master of Science. The work was supported by the award of a Frederick Clifford Scholarship.

(2) The author wishes to express his thanks and indebtedness to Dr. H. Burkill for indicating the direction of this investigation and for his many helpful suggestions. In particular, it should be mentioned that Dr. Burkill was well aware of the potency of the condition $\lambda_{n+1}-\lambda_{n}$ $\geqq l>0$. 
In the present paper, however, attention is confined to series having sumfunctions integrable in the sense of Lebesgue, and to the $U, S^{p}, W^{p}, B^{p}$ types of almost periodicity. In Theorem I the same restrictions are imposed on the $\lambda_{n}$ as in Burkill's theorem. The conditions imposed on $f(x)$ are, in a sense, uniform restrictions on properties possessed by any $L$-integrable function over a finite interval. It is found that the hypothesis of convergence can be relaxed in an enumerable set. Theorem II embodies another result for bounded, uniformly continuous sum-functions, showing that the condition $\lambda_{n+1}-\lambda_{n}$ $\geqq l>0$ can be replaced by the less restrictive condition

$$
\sum_{n=1}^{\infty} \lambda_{n}^{-2}<\infty
$$

when the series converges everywhere.

2. Notation. The notation used for distance-functions is that commonly employed in the literature (e.g. see Besicovitch [2]).

The symbol $D_{G^{p}}$, is used to denote any one of the distance-functions $D_{B^{p}}, D_{W^{p}}, D_{S^{p}}(p \geqq 1)$.

For $n \geqq 1$, we write

$$
\begin{aligned}
& A_{n}(x)=a_{n} \cos \lambda_{n} x+b_{n} \sin \lambda_{n} x \\
& B_{n}(x)=\frac{b_{n}}{\lambda_{n}} \cos \lambda_{n} x-\frac{a_{n}}{\lambda_{n}} \sin \lambda_{n} x \\
& \Phi(x)=-\sum_{n=1}^{\infty} \lambda_{n}^{-2} A_{n}(x)
\end{aligned}
$$

We also define

$$
\begin{gathered}
\Delta_{h}^{2} g(x)=g(x+h)+g(x-h)-2 g(x), \\
\bar{D}_{2} g(x)=\limsup _{h \rightarrow 0}\left[h^{-2} \Delta_{h}^{2} g(x)\right], \quad \hat{D}_{2} g(x)=\liminf _{h \rightarrow 0}\left[h^{-2} \Delta_{h}^{2} g(x)\right],
\end{gathered}
$$

and write

$$
D_{2} g(x)=\lim _{h \rightarrow 0}\left[h^{-2} \Delta_{h}^{2} g(x)\right]
$$

whenever the limit exists.

3. Theorem I. If $\left(\lambda_{n}\right)$ is a sequence of real numbers such that

$$
\lambda_{1}>0 \quad \text { and } \quad \lambda_{n+1}-\lambda_{n} \geqq l>0 \quad(n=1,2, \cdots)
$$

and if the series 


$$
a_{0} / 2+\sum_{n=1}^{\infty}\left(a_{n} \cos \lambda_{n} x+b_{n} \sin \lambda_{n} x\right)
$$

converges, except possibly in an enumerable set $E$, to a function $f(x)$ belonging to the class $L^{p}(p \geqq 1)$ on any finite interval and such that

$$
\lim _{\delta \rightarrow 0}\left[\sup _{-\infty<x<\infty} \int_{x}^{x+\delta} f(t) d t\right]=0
$$

and

$$
\lim _{\delta \rightarrow 0} D_{G^{p}}[f(x+\delta), f(x)]=0
$$

where $G^{p}$ is one of $B^{p}, W^{p}, S^{p}$, then $f(x)$ is $G^{p}$-a.p. and the given series is the Fourier series of $f(x)$.

In particular, if $f(x)$ is bounded and uniformly continuous, then it is u.a.p.

Before proceeding to the proof of Theorem 1, it is convenient to establish a number of lemmas. Where these are simple extensions of well-known results $\left({ }^{3}\right)$, they are stated without proof.

LEMMA 1. If $\lambda_{n} \rightarrow \infty$ as $n \rightarrow \infty$, and if $a_{n} \cos \lambda_{n} x+b_{n} \sin \lambda_{n} x \rightarrow 0$ in a set $E$ of positive measure, then $a_{n} \rightarrow 0$ and $b_{n} \rightarrow 0$.

Definition $\mathrm{I}\left({ }^{4}\right)$. A series $\sum_{n=0}^{\infty} u_{n}$ will be said to be "summable $\left(R, \lambda_{n}\right)$ to $S^{\prime \prime}$ if

$$
u_{0}+\sum_{n=1}^{\infty} u_{n}\left(\frac{\sin \lambda_{n} h}{\lambda_{n} h}\right)^{2} \rightarrow S
$$

as $h \rightarrow 0$, where $0<\lambda_{1}<\lambda_{2}<\cdots<\lambda_{n}<\cdots$ and $\lambda_{n} \rightarrow \infty$ as $n \rightarrow \infty$.

Lемма $2\left({ }^{4}\right)$. The method $\left(R, \lambda_{n}\right)$ is regular; i.e. if a series converges, it is summable $\left(R, \lambda_{n}\right)$ to the same sum. then

Lemma 3. If $\sum_{n=1}^{\infty} \lambda_{n}^{-2}<\infty$, and $x$ is a point of convergence of $\sum_{n=1}^{\infty} A_{n}(x)$,

$$
D_{2} \Phi(x)=\lim _{h \rightarrow 0} \sum_{n=1}^{\infty} A_{n}(x)\left(\frac{\sin \lambda_{n} h}{\lambda_{n} h}\right)^{2}=\sum_{n=1}^{\infty} A_{n}(x) .
$$

LemMA 4. If $\lambda_{1}>0$ and $\lambda_{n+1}-\lambda_{n} \geqq l>0 \quad(n=1,2, \cdots)$, and $u_{n} \rightarrow 0$, as $n \rightarrow \infty$, then

( ${ }^{3}$ See, for example, Hardy and Rogosinski [6].

(4) H. Burkill [4] defines summability $\left(R, \lambda_{n}\right)$ and establishes Lemma 2 with the condition $\lambda_{n+1}-\lambda_{n} \geqq l>0$. 


$$
h \sum_{n=1}^{\infty} u_{n}\left(\frac{\sin \lambda_{n} h}{\lambda_{n} h}\right)^{2} \rightarrow 0
$$

as $h \rightarrow 0$.

Definition 2. A function $g(x)$ is said to be "smooth at $x$ " if

$$
\Delta_{h}^{2} g(x)=o(h)
$$

as $h \rightarrow 0$.

LemMA 5. If $\lambda_{n+1}-\lambda_{n} \geqq l>0$ and if $\sum_{n=1}^{\infty} A_{n}(x)$ converges in a set $E$ of positive measure, then

$$
\Phi(x)=-\sum_{n=1}^{\infty} \lambda_{n}^{-2} A_{n}(x)
$$

is smooth at any point $x$ of $E$.

Lemma 6. Suppose that $f(x)$ is finite except in an enumerable set $E$, and integrable in $(a, b)$; that $g(x)$ is continuous and smooth, and that

$$
\hat{D}_{2} g(x) \leqq f(x) \leqq \bar{D}_{2} g(x)
$$

except in E. Then

$$
g(x)-\int_{a}^{x} d t \int_{a}^{t} f(u) d u
$$

is linear in $(a, b)$.

LEMma 7. If $0<\lambda_{1}<\lambda_{2}<\cdots<\lambda_{n}<\cdots$ and $\lambda_{n+1}-\lambda_{n} \geqq l>0$, and if $a_{n}=o\left(\lambda_{n}^{-1}\right), b_{n}=o\left(\lambda_{n}^{-1}\right)$, then the series

$$
(1 / 2) a_{0}+\sum_{n=1}^{\infty}\left(a_{n} \cos \lambda_{n} x+b_{n} \sin \lambda_{n} x\right)
$$

converges almost everywhere to an $S^{2}$ a.p. function $f(x)$ of which it is the Fourier series.

If, moreover, $a_{0}=0$, then the series integrated term-by-term converges to a u.a.p. function $F(x)$, where

$$
F(x)=\int^{x} f(t) d t
$$

A proof of Lemma 7, based on results due to Titchmarsh [7] and Bochner [8] has been given by $\mathrm{H}$. Burkill [4].

Proof of Theorem 1. Put 


$$
F(x)=f(x)-a_{0} / 2=\sum_{n=1}^{\infty} A_{n}(x) .
$$

By Lemma $1, a_{n}=o(1), b_{n}=o(1)$, so that the coefficients in the series $-\sum_{n=1}^{\infty} B_{n}(x)$ satisfy the conditions of Lemma 7 . Hence $-\sum_{n=1}^{\infty} B_{n}(x)$ converges almost everywhere to an $S^{2}$ a.p. function $\phi(x)$ of which it is the Fourier series; and furthermore the series $-\sum_{n=1}^{\infty} \lambda_{n}^{-2} A_{n}(x)$ converges uniformly to a u.a.p. function $\Phi(x)$, where

$$
\Phi(x)=\int^{x} \phi(t) d t
$$

By Lemma 5, $\Phi(x)$ is everywhere smooth; and by Lemma 3, $D_{2} \Phi(x)=F(x)$ except possibly in the at most enumerable set $E$ in which $\sum_{n=1}^{\infty} A_{n}(x)$ diverges. Thus we may take $g(x)=\Phi(x)$ in Lemma 6 , so that if $-m<x<m$,

$$
\Phi(x)=\int_{-m}^{x} d t \int_{-m}^{t} F(u) d u=C_{m} x+D_{m}
$$

where $C_{m}, D_{m}$ are constants whose value may depend on $m$. Therefore

$$
\Phi^{\prime}(x)-\int_{-m}^{x} F(t) d t=C_{m} .
$$

But from (1),

$$
\Phi^{\prime}(x)=\phi(x) \text { p.p. }
$$

and so may we take

$$
\phi(x)=\int^{x} F(t) d t+K_{m}
$$

everywhere in $(-m, m)$, since the value of $\phi(x)$ may be modified if necessary in a set of zero measure without affecting its $S^{2}$ almost periodicity. Hence, for any $x$ in $(-m, m)$

$$
|\phi(x+\delta)-\phi(x)|=\left|\int_{x}^{x+\delta} F(t) d t\right| .
$$

This equation is independent of $m$, so it holds for $-\infty<x<\infty$; and since

$$
\int_{x}^{x+\delta} F(t) d t=\int_{x}^{x+\delta} f(t) d t-(\delta / 2) a_{0},
$$

the condition imposed on $\int_{x}^{x+\delta} f(t) d t$ as $\delta \rightarrow 0$ implies

$$
\lim _{\delta \rightarrow 0} \sup |\phi(x+\delta)-\phi(x)|=0 \text {. }
$$


Thus $\phi(x)$ is a uniformly continuous $S$ a.p. function and hence, by a theorem of Bochner [8], it is u.a.p.: so also, therefore, is the function

$$
f_{\eta}(x)=\frac{1}{\eta}[\phi(x+\eta)-\phi(x)]+a_{0} / 2=\frac{1}{\eta} \int_{x}^{x+\eta} f(t) d t,
$$

for any $\eta>0$.

To prove that $f(x)$ is $G^{p}$ a.p. it is sufficient to show that

$$
\lim D_{\sigma^{p}}\left[f(x), f_{\eta}(x)\right]=0,
$$

and we now prove that this condition is satisfied when $f(x)$ is " $G^{p}$-continuous," i.e. when $\lim _{\delta \rightarrow 0} D_{G^{p}}[f(x+\delta), f(x)]=0(p \geqq 1)$. The proof is written down only for $G^{p} \equiv B^{p}$; the results corresponding to $G^{p} \equiv W^{p}, S^{p}$, respectively can be obtained similarly.

Since $f(x)$ is $B^{p}$-continuous, given $\epsilon>0$ we can find $T_{0}=T_{0}(\epsilon)>0$ such that

$$
0 \leqq\left[\frac{1}{T} \int_{0}^{T}|f(x+\delta)-f(x)|^{p} d x\right]^{1 / p} \leqq D_{B p}[f(x+\delta), f(x)]+\epsilon
$$

for all $T \geqq T_{0}$, and a number $\delta_{0}=\delta_{0}(\epsilon)>0$ such that for $|\delta| \leqq \delta_{0}$,

$$
D_{B}^{p}[f(x+\delta), f(x)] \leqq \epsilon ;
$$

therefore, if $T \geqq T_{0}$ and $|\delta| \leqq \delta_{0}$,

$$
\left[\frac{1}{T} \int_{0}^{T}|f(x+\delta)-f(x)| p d x\right]^{1 / p} \leqq 2 \epsilon .
$$

Now, by an application of Minkowski's inequality, we have

$$
\begin{aligned}
{\left[\frac{1}{T} \int_{0}^{T} \mid f(t)-\frac{1}{\eta}\right.} & \left.\left.\int_{t}^{t+\eta} f(u) d u\right|^{p} d t\right]^{1 / p} \\
\leqq & {\left[\frac{1}{T} \int_{0}^{T}\left|f\left(t+\delta_{0}\right)-f(t)\right|^{p} d t\right]^{1 / p} } \\
& +\left[\frac{1}{T} \int_{0}^{T}\left\{\frac{1}{\eta} \int_{0}^{\eta}\left|f(t+u)-f\left(t+\delta_{0}\right)\right| d u\right\}^{p} d t\right]^{1 / p} \\
= & {\left[I_{1}\left(T, \delta_{0}\right)\right]^{1 / p}+\left[\frac{1}{T} \int_{0}^{T}\left\{I_{2}\left(t, \delta_{0}\right)\right\} p d t\right]^{1 / p} }
\end{aligned}
$$

say. Here

$$
\left[I_{1}\left(T, \delta_{0}\right)\right]^{1 / p} \leqq 2 \epsilon
$$

when $T \geqq T_{0}$; also, by an application of Hölder's inequality, we have 


$$
\begin{aligned}
I_{2}\left(t, \delta_{0}\right) & \equiv \frac{1}{\eta} \int_{0}^{\eta}\left|f(t+u)-f\left(t+\delta_{0}\right)\right| d u \\
& \leqq \frac{1}{\eta}\left[\int_{0}^{\eta}\left|f(t+u)-f\left(t+\delta_{0}\right)\right| p d u\right]^{1 / p} \cdot\left[\int_{0}^{\eta} d u\right]^{(p-1) / p} \\
& =\eta^{-1 / p}\left[\int_{0}^{\eta}\left|f(t+u)-f\left(t+\delta_{0}\right)\right| p d u\right]^{1 / p}
\end{aligned}
$$

so that

$$
\begin{aligned}
& \frac{1}{T} \int_{0}^{T}\left\{I_{2}\left(t, \delta_{0}\right)\right\}^{p} d t \leqq \frac{1}{T} \int_{0}^{T}\left\{\frac{1}{\eta} \int_{0}^{\eta}\left|f(t+u)-f\left(t+\delta_{0}\right)\right|^{p} d u\right\} d t \\
& =\frac{1}{\eta} \int_{0}^{\eta}\left[\left\{\left(1+\frac{u}{T}\right)(T+u)^{-1} \int_{0}^{T+u}-\frac{1}{T} \int_{0}^{u}\right\}\left|f(t)-f\left(t+\delta_{0}-u\right)\right|^{p} d t\right] d u \\
& \leqq \frac{1}{\eta} \int_{0}^{\eta}\left\{\left(1+\frac{\eta}{T}\right)(2 \epsilon)^{p} d u+\frac{1}{T} \int_{0}^{\eta}\left|f(t)-f\left(t+\delta_{0}-u\right)\right|^{p} d t\right\} d u
\end{aligned}
$$

when $T \geqq T_{0}$ and $0<\eta \leqq 2 \delta_{0}$,

$$
\begin{array}{ll}
\leqq\left(1+\frac{2 \delta_{0}}{T}\right)(2 \epsilon)^{p}+\sup _{0 \leqq u \leqq 2 \delta_{0}}\left\{\frac{1}{T} \int_{0}^{2 \delta_{0}}\left|f(t)-f\left(t+\delta_{0}-u\right)\right| p d t\right\} \\
\leqq(1+\epsilon)(2 \epsilon)^{p}+\epsilon^{p}<(5 \epsilon)^{p} & (0<
\end{array}
$$

for $T>\max \left(T_{0}, T_{1}\right)$, where $T_{1}=T_{1}\left(\epsilon, \delta_{0}\right)=T_{1}(\epsilon)$. Hence, from (2)-(4)

$$
\begin{aligned}
D_{B^{p}}\left[f(x), f_{\eta}(x)\right] & =\lim _{T \rightarrow \infty}\left[\frac{1}{T} \int_{0}^{T}\left|f(t)-\frac{1}{\eta} \int_{t}^{t+\eta} f(u) d u\right|^{p} d t\right]^{1 / p} \\
& <2 \epsilon+5 \epsilon=7 \epsilon \quad(0<\epsilon<1)
\end{aligned}
$$

when $0<\eta \leqq 2 \delta_{0}(\epsilon)$. Since $\epsilon$ is arbitrarily small, it follows that

$$
\lim _{\eta \rightarrow 0} D_{B^{p}}\left[f(x), f_{\eta}(x)\right]=0 \text {; thus } f(x) \text { is } B^{p} \text { a.p. }
$$

Furthermore, by integration by parts, we have for any real value of $\lambda$,

$$
\begin{aligned}
\frac{1}{T} \int_{0}^{T} F(x) \cos \lambda x d x & =\frac{1}{T}[\phi(x) \cos \lambda x]_{0}^{T}+\frac{\lambda}{T} \int_{0}^{T} \phi(x) \sin \lambda x d x \\
& \rightarrow \lambda M[\phi(x) \sin \lambda x]
\end{aligned}
$$

as $T \rightarrow \infty$, since $\phi(x)$ is bounded. Since $-\sum_{n=1}^{\infty} B_{n}(x)$ is the Fourier series of $\phi(x)$,

$$
M[\phi(x) \sin \lambda x]= \begin{cases}0 & \text { if } \lambda \neq \lambda_{n}, \\ a_{n} \lambda_{n}^{-1} & \text { if } \lambda=\lambda_{n} .\end{cases}
$$


Hence

$$
M\left[\left(f(x)-a_{0} / 2\right) \cos \lambda x\right]= \begin{cases}0 & \text { if } \lambda \neq \lambda_{n} \\ a_{n} & \text { if } \lambda=\lambda_{n}\end{cases}
$$

But

$$
M\left[\left(a_{0} / 2\right) \cos \lambda x\right]= \begin{cases}0 & \text { if } \lambda \neq 0 \\ a_{0} / 2 & \text { if } \lambda=0\end{cases}
$$

therefore

$$
M\lfloor f(x) \cos \lambda x\rfloor=\left\{\begin{array}{lr}
a_{0} / 2 & \text { if } \lambda=0, \\
a_{n} & \text { if } \lambda=\lambda_{n}(n=1,2, \cdots), \\
0 & \text { for all other } \lambda .
\end{array}\right.
$$

Similarly,

$$
M[f(x) \sin \lambda x]=\left\{\begin{array}{lr}
b_{n} & \text { if } \lambda=\lambda_{n}(n=1,2, \cdots), \\
0 & \text { for all other } \lambda .
\end{array}\right.
$$

These show that the given series $a_{0} / 2+\sum_{n=1}^{\infty}\left(a_{n} \cos \lambda_{n} x+b_{n} \sin \lambda_{n} x\right)$ is the Fourier series of its sum-function $f(x)$.

If $f(x)$ is continuous at $x$, then

$$
\int_{x}^{x+\delta} f(t) d t=\delta \cdot f(x)+o(\delta)
$$

as $\delta \rightarrow 0$, so that if $f(x)$ is everywhere continuous, the condition

$$
\lim _{\delta \rightarrow 0} \sup _{-\infty<x<\infty}\left|\int_{x}^{x+\delta} f(t) d t\right|=0
$$

is plainly equivalent to the condition that $f(x)$ be everywhere bounded. Also, uniform continuity of $f(x)$ for $-\infty<x<\infty$ is the same as $\lim _{\delta \rightarrow 0} D_{U}[f(x+\delta), f(x)]=0$. Thus if $f(x)$ is bounded and uniformly continuous for $-\infty<x<\infty$, the function $\phi(x)$ is u.a.p. and has the Fourier series $-\sum_{n=1}^{\infty} B_{n}(x)$ as before; furthermore, $f_{\eta}(x)$ is u.a.p. and

$$
\begin{aligned}
f_{\eta}(x) & =\eta^{-1}[\phi(x+\eta)-\phi(x)]+a_{0} / 2=f(x+\theta \eta) \quad(0<\theta<1) \\
& \rightarrow f(x)
\end{aligned}
$$

uniformly for $-\infty<x<\infty$ as $\eta \rightarrow 0$, which proves that $f(x)$ is u.a.p. It can be shown as before that $a_{0} / 2+\sum_{n=1}^{\infty} A_{n}(x)$ is the Fourier series of $f(x)$.

4. However, when $f(x)$ is bounded and uniformly continuous, and the series converges everywhere to $f(x)$, the condition imposed on the $\lambda_{n}$ in 
Theorem 1 is unnecessarily restrictive and can be replaced by the condition $\sum_{n=1}^{\infty} \lambda_{n}^{-2}<\infty$.

THEOREM 2. If $\left(\lambda_{n}\right) n=1,2, \cdots$ is a sequence of real numbers such that $0<\lambda_{1}<\lambda_{2}<\cdots<\lambda_{n}<\cdots$ and $\sum_{n=1}^{\infty} \lambda_{n}^{-2}<\infty$, and if for all $x$, the series

$$
a_{0} / 2+\sum_{n=1}^{\infty}\left(a_{n} \cos \lambda_{n} x+b_{n} \sin \lambda_{n} x\right)
$$

converges to a bounded, uniformly continuous function $f(x)$, then $f(x)$ is u.a.p. and this series is the Fourier series of $f(x)$.

The proof of Theorem 2 requires an additional lemma $\left({ }^{5}\right)$, viz.,

Lemma 8. If, for $-\infty<x<\infty, g(x)$ is continuous and $\bar{D}_{2} g(x) \geqq c$, then

$$
h^{-2} \Delta_{h g}^{2}(x) \geqq c ;
$$

and if $\hat{D}_{2} g(x) \leqq c$, then $h^{-2} \Delta_{h}^{2} g(x) \leqq c$.

Proof of Theorem 2. Write

$$
\Psi(x)=(1 / 4) a_{0} x^{2}+\Phi(x) .
$$

Since $\sum_{n=1}^{\infty} \lambda_{n}^{-2}<\infty$ and $a_{n}, b_{n} \rightarrow 0$ by Lemma 1 , the series defining $\Phi(x)$ converges uniformly and absolutely for all $x$; hence $\Psi(x)$ is continuous, and for $h>0$,

$$
f_{h}(x)=\frac{\Delta_{2 h}^{2} \Psi(x)}{4 h^{2}}=\frac{a_{0}}{2}+\sum_{n=1}^{\infty} A_{n}(x)\left(\frac{\sin \lambda_{n} h}{\lambda_{n} h}\right)^{2} .
$$

Here, the series converges uniformly for all $x$, so that for any $h>0$ the function $f_{h}(x)$ is u.a.p. By Lemma $2, f_{h}(x) \rightarrow f(x)$ as $h \rightarrow 0$ for all $x$, i.e. $D_{2} \Psi(x)$ $=f(x)$, and so $\left|D_{2} \Psi(x)\right| \leqq K$, where $K$ is the upper bound of $|f(x)|$; hence by Lemma $8,\left|f_{h}(x)\right| \leqq K$.

Consider now the function

$$
f_{h}(x, \lambda)=\frac{1}{T} \int_{-\infty}^{\infty} f_{h}(x+2 t) \frac{\sin ^{2} \lambda t}{\lambda t^{2}} d t
$$

where $h>0$ and $\lambda>0$, together with the formula

$$
\frac{1}{\pi} \int_{-\infty}^{\infty} e^{2 \mu i t} \frac{\sin ^{2} \lambda t}{\lambda t^{2}} d t=\left\{\begin{array}{lr}
1-|\mu| / \lambda & \text { for } 0 \leqq|\mu| \leqq \lambda, \\
0 & \text { for } \lambda<|\mu| .
\end{array}\right.
$$

Since the sequence of trigonometric polynomials

$$
\frac{a_{0}}{2}+\sum_{n=1}^{N} A_{n}(x)\left(\frac{\sin \lambda_{n} h}{\lambda_{n} h}\right)^{2} \quad(N=1,2 \cdots)
$$

(6) Again, a simple generalization of a well-known result; see Hardy and Rogosinski [6]. 
converges uniformly to $f_{h}(x)$ as $N \rightarrow \infty$, it is easy to show by use of the above formula that

$$
f_{h}(x, \lambda)=\frac{a_{0}}{2}+\sum_{\lambda_{n}<\lambda}\left(1-\frac{\lambda_{n}}{\lambda}\right)\left(\frac{\sin \lambda_{n} h}{\lambda_{n} h}\right)^{2} A_{n}(x),
$$

which is a trigonometric polynomial. Hence the function

$$
\lim _{h \rightarrow 0} f_{h}(x, \lambda)=\frac{a_{0}}{2}+\sum_{\lambda_{n}<\lambda}\left(1-\frac{\lambda_{n}}{\lambda}\right) A_{n}(x) \equiv f(x, \lambda)
$$

say is u.a.p.

To prove the theorem, it is now sufficient to show that $f(x, \lambda) \rightarrow f(x)$ uniformly in $x$ as $\lambda \rightarrow \infty$. Since $\left|f_{h}(x)\right| \leqq K$ everywhere, for all values of $h>0$, the integrand in (5) is uniformly bounded and is $O\left(1 / \lambda t^{2}\right)$, uniformly in $x$ and $h$, for large $t$. Hence

$$
f_{h}(x, \lambda)=\frac{1}{\pi} \int_{-T}^{T} f_{h}(x+2 t) \frac{\sin ^{2} \lambda t}{\lambda t^{2}} d t+O\left(\frac{1}{\lambda T}\right),
$$

whence, by an application of Lebesgue's theorem on bounded convergence, and then letting $T \rightarrow \infty$, we find

$$
f(x, \lambda)=\frac{1}{\pi} \int_{-\infty}^{\infty} f(x+2 t) \frac{\sin ^{2} \lambda t}{\lambda t^{2}} d t .
$$

Further, since $f(x)$ is uniformly continuous, given $\epsilon>0$ we can find $\lambda_{0}=\lambda_{0}(\epsilon)$ $>\epsilon^{-2}$ such that for $\lambda \geqq \lambda_{0}$ and all $x$,

$$
|f(x+2 t)-f(x)| \leqq \epsilon
$$

whenever $0 \leqq 2 t \leqq \lambda^{-1 / 2}$. Therefore, for $\lambda \geqq \lambda_{0}$ and all $x$, we have for any number $\epsilon$ in $(0,1)$,

$$
\begin{aligned}
\dot{\pi}^{\prime} & f(x, \lambda)-f(x)|=| \int_{0}^{\infty}\{f(x+2 t)+f(x-2 t)-2 f(x)\} \frac{\sin ^{2} \lambda t}{\lambda t^{2}} d t \mid \\
\leqq & \left(\int_{0}^{\lambda^{-1}}+\int_{\lambda^{-1}}^{\lambda^{-1 / 2}}+\int_{\lambda^{-1 / 2}}^{\infty}\right)\{|f(x+2 t)-f(x)|+|f(x)-f(x-2 t)|\} \\
& \quad \frac{\sin ^{2} \lambda t}{\lambda t^{2}} d t \\
\leqq & \int_{0}^{\lambda^{-1}} 2 \lambda \epsilon d t+2 \epsilon\left[\frac{-1}{\lambda t}\right]_{\lambda^{-1}}^{\lambda^{-1 / 2}}+4 K\left[\frac{-1}{\lambda_{t}}\right]_{\lambda^{-1 / 2}}^{\infty} \\
= & 2 \epsilon+2 \epsilon\left(1-\lambda^{-1 / 2}\right)+4 K / \lambda^{-1 / 2} \\
< & 4(K+1) \epsilon .
\end{aligned}
$$


Therefore $f(x)$ is u.a.p. and its Fourier series is the formal limit of the series (6) as $\lambda \rightarrow \infty$, viz., $a_{0} / 2+\sum_{n=1}^{\infty} A_{n}(x)$.

\section{BIBLIOGRAPHY}

1. A. S. Besicovitch, On generalized almost periodic functions, Proc. London Math. Soc. vol. 26 (1927) pp. 495-512.

2. - Almost periodic functions, Cambridge, University Press, 1932.

3. H. Burkill, Almost periodicity and non-absolutely integrable functions, Proc. London Math. Soc. vol. 53 (1951) pp. 32-42.

4. - Cesdro-Perron almost periodic functions, Proc. London Math. Soc. vol. 2 (1952) pp. 150-174.

5. J. C. Burkill, Integrals and trigonometric series, Proc. London Math. Soc. vol. 2 (1952) pp. $46-57$.

6. G. H. Hardy and W. W. Rogosinski, Fourier series, Cambridge Tract No. 38 (1944) Chapter VII, pp. 83-92.

7. E. C. Titchmarsh, A class of trigonometric series, J. London Math. Soc. vol. 3 (1928) pp. 300-304.

8. S. Bochner, Properties of Fourier series of almost periodic functions, Proc. London Math. Soc. vol. 26 (1927) pp. 433-452.

\section{UNIVERSITY OF SHEFFIELD,}

SHEFFIELD, ENGLAND 